\title{
Effect of xylitol-containing carbohydrate mixtures on acid and ammonia production in suspensions of salivary sediment
}

\author{
EVA SÖDERLING', JUHA TALONPOIKA' AND KAUKO K MÄKINEN² \\ 'Institute of Dentisiry, University of Turku, Turku, Finland and 'School of Dentistry, The University of Michigan, \\ Ann Artor, Michigan, USA
}

\begin{abstract}
Söderling E, Talonpoika J, Mäkinen KK: Effect of xylitol-containing carbohydrate mixtures on acid and aramonia production in suspensions of salivary sediment. Scand $\mathbb{J}$ Dent Res 1987; 95 : $405-10$.
\end{abstract}

\begin{abstract}
H}$ changes and the production of lactic acid, acetic acid and ammonia were studied in suspensions of salivary sediment supplemented with mixtures of xylitol and other carbohydrate sweeteners. The only mixtures which increased the $\mathrm{pH}$ values of the suspensions were those containing xylitol alone or mixtures of xylitol and sorbitol. Mixtures of xylitol and Lycasin 80/ 55 caused a relatively small $\mathrm{pH}$ reduction. Xylitol was not able to inhibit the acid production from the easily fermented glucose, fructose and Lycasin 05/60. The levels of lactic acid, determined in the incubation mixtures, directly reflected these $\mathrm{pH}$ changes. The levels of aceic acid and ammonia were, however, relatively similar in all incubation mixtures. The results suggest that the inhibitory effects of xylitol on acid production of oral flora should be retained, provided that xylitol is used either alone or in mixtures with slowly fermentable carbohydrates, such as sorbitol and Lycasin 80/55.
\end{abstract}

Key words: acid production; carbohydrates; dental plaque; sweeteners; xylitol.

Eva Söderling, School of Dentistry, Department of Oral Biology, University of Michigan, Ann Arbor, Michigan 48109, USA.

Accepted for publication 24 January 1987.

The majority of oral bacteria lack the ability to ferment xylitol (1-4). The inability of plaque to metabolize xylitol may be one of the factors leading to a reduction both in the amount of plaque and the incidence of Streptococcus mutans, both of which have been found to result from between-meal intake of xylitol $(5,6)$. The enhancement of reminerallization of carious lesions observed in connection with the intake of xylitol seems to be based on the above microbiologic effects and the ability of this polyol to stimulate the flow of saliva without a $\mathrm{pH}$ decrease (7). Thus, the nonfermentability and nonacidogenicity of xylitol seem to be behind most of its favorable actions in the oral cavity (8).

Although the beneficial oral effects of xyli- 
tol are widely acknowledged, the fact remains that xylitol, partly as a result of its high price and partly as a result of food technologic aspects, is normally used in mixtures with other sweeteners. Only a relatively small number of studies have, however, dealt with the dental effects of mixtures of xylitol and other carbohydrates. The aim of the present study was to elucidate the effects of various mixtures of xylitol and other common carbohydrate sweeteners on the acid and ammonia production by salivary sediment.

\section{Material and methods}

Carbohydrates - The following carbohydrates were used: xylitol (Finnish Sugar Co., Helsinki, Finland), fructose, glucose, sorbitol (Sigma Chemical Co., St. Louis, MO, USA), Lycasin 05/60 and Lycasin 80/55 (Raquette, Lille, France). Xylitol (x) and the other carbohydrates (ch) were used in the following ratios: $\mathrm{x} / \mathrm{ch}=0 / 100 ; 50 / 50 ; 99 / 1$; $99.5 / 0.5 ; 100 / 0$ (wt/wt).

Salivary sediment mixture - The salivary sediment incubations were performed according to $\mathrm{K}_{\text {LEIN- }}$ BERG (9). Whole saliva samples were collected from dental students (five women and five men, aged $2 \mathrm{I}-23 \mathrm{yr}) 2 \mathrm{~h}$ after breakfast, using $10 \mathrm{~min}$ paraffin wax stimulation. The samples were pooled and divided into $10-\mathrm{ml}$ aliquots. The aliquots were centrifuged $\left(12000 \mathrm{xg}, 10 \mathrm{~min},+4^{\circ} \mathrm{C}\right)$ and the supernatant fluids and the pellets stored separately at $-20^{\circ} \mathrm{C}$ until used in the incubation experiments within $4 \mathrm{wk}$.

Incubation conditions and assay methods - Before the incubation each sediment was washed with $10 \mathrm{ml}$ of $0.9 \% \mathrm{NaCl}$ (10-s Vortex agitation, followed by a 5 -s sonication and centrifugation for $10 \mathrm{~min}$ at $\left.12000 \mathrm{xg},+4{ }^{\circ} \mathrm{C}\right)$. The washed sediments were combined with $4 \mathrm{ml}$ of Millipore-filtered (HAWP $0.45 \mu \mathrm{m}$ ) saliva supernatant. These mixtures were homogenized using Vortex treatmert and sonification as above. $300 \mu \mathrm{l}$ aliquots of the suspensions were mixed with $300 \mu \mathrm{l}$ of a $20 \%(w / v)$ carbohydrate solution (mixtures of xylitol and other carbohydrates mentioned above). The resulting suspensions were incubated for $4 \mathrm{~h}$ at $37^{\circ} \mathrm{C}$. The final total concentration of the carbohydrates in the incubation mixtures was thus $10 \%(\mathrm{w} / \mathrm{v})$. For Lycasin $80 / 55$ the amount of dry matter in the syrup was used as a basis for calculations. The $\mathrm{pH}$ values were measured electrometrically at the beginning and after the 4-h incubations. After the incubation, the mixtures were centrifuged as above and the supernatants stored at $-20^{\circ} \mathrm{C}$ for the determination of lactic acid, acetic acid (using the Boehringer-Mannheim test kits) and ammonia (10).

\section{Results}

Fig. 1 illustrates the $\mathrm{pH}$ changes measured for the various $\mathrm{x} / \mathrm{ch}$ mixtures. The $\mathrm{pH}$ drops caused by $10 \%$ fructose, glucose or Lycasin $05 / 60$ alone $(\mathrm{x} / \mathrm{ch}=0 / 100)$ were large and of almost identical magnitude. For $10 \%$ sorbitol, Lycasin 80/55, and the control containing water instead of added carbohydrates, slight decreases in the $\mathrm{pH}$ values were observed. The presence of $10 \%$ xylitol alone, however, resulted in a slight $\mathrm{pH}$ increase.

The mixtures of fructose or glucose with xylitol behaved identically. The decreases in the $\mathrm{pH}$ values directly reflected the amounts of fermentable fructose or glucose present in these incubation mixtures. The presence of various amounts of xylitol had no or only a slight effect on the decrease of the $\mathrm{pH}$ values. With these two carbohydrates, the only mixture which did not show a large $\mathrm{pH}$ drop was the $x / c h=0.5 / 99.5$ mixture. The mixture $x /$ $\mathrm{ch}=50 / 50$ of Lycasin $05 / 60$ and xylitol decreased the $\mathrm{pH}$ values similarly to the corresponding mixtures of fructose or glucose with xylitol. For the $\mathrm{x} / \mathrm{ch}=99 / 1 \mathrm{mix}$ tures of Lyca$\sin 05 / 60$ and xylitol, however, a smaller $\mathrm{pH}$ drop was observed as compared to fructose or glucose. With all combinations of sorbitol and xylitol not an increase but a decrease in the $\mathrm{pH}$ values was observed. Mixtures of Lycasin 80/55 and xylitol showed a relatively small pH drop.

The amount of lactic acid produced during the incubations reflected the results observed in the $\mathrm{pH}$ measurements (Fig. 2). The lowest amounts of acetic acid were, however, observed in mixtures containing xylitol as the only added carbohydrate. The increase. 


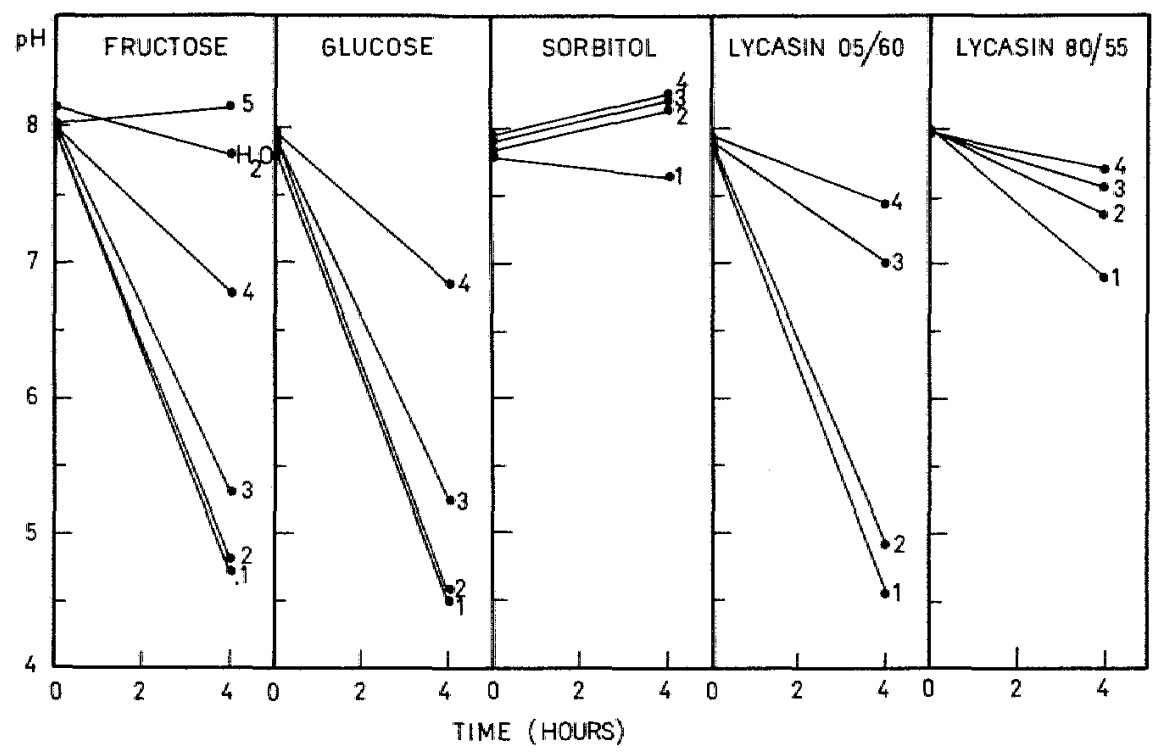

Fig. I. pH-changes in suspensions of salivary sediment for $10^{0} / 0,(\mathrm{w})$ mixtures of xylitol ( $\mathrm{x}$ ) and other carbohydrates (ch). The pH measurements were made at the start of the incubation, and after $4 \mathrm{~h}$. 1 : $\mathrm{x} / \mathrm{ch}=0 / 100 ; 2: \mathrm{x} / \mathrm{ch}=50 / 50 ; 3: \mathrm{x} / \mathrm{ch}=99 / 1 ; \mathrm{x} / \mathrm{ch}=99.5 / 0.5 ; 5 ; \mathrm{x} / \mathrm{ch}=100 / 0$.

in the relative amount of xylitol in the sorbitol-xylitol mixtures resulted in decreased amounts of acetic acid. This effect exerted by xylitol was also observed with mixtures of xylitol and Lycasin $80 / 55$ (not shown), but not with the other $\mathrm{x} / \mathrm{ch}$ mixtures studied. The acetic acid levels of the control mixtures incubated for $4 \mathrm{~h}$ in an ice-bath showed values which were about half of those found after incubation at $37^{\circ} \mathrm{C}$ (not shown).

Changes in the $x / c h$ ratio had no clear effect on the amounts of ammonia produced (Fig. 2). The ammonia levels of the controls kept in an ice-bath were about $50 \%$ lower than those found after incubation at $37^{\circ} \mathrm{C}$ (not shown). The presence of fructose seemed to result in a lower production of ammonia, the ammonia levels detected were the smallest in the presence of fructose only. The production of ammonia increased when the relative portion of fructose was decreased in the $x / c h$ mixtures. The acid and ammonia levels observed in the presence of mixtures of xylitol and Lycasin 80/55 closely resembled those mixtures of xylitol and sorbitol; the levels of lactic acid were only slightly higher in the former.

\section{Discussion}

Several studies have demonstrated that xylitol does not cause any pH-reduction in plaque either in viwo or in vitro $(2,1 \mathbb{1}-13)$. In pure cultures, xylitol inhibits the growth and acid production of some oral bacteria $(14,15)$. The results of studies on acid production with mixtures of xylitol and other carbohydrates are partly controversial, however $(12,16-20)$. For example, xylitol decreased the acid production in plaque when used simultaneously with glucose or fructose (16). Other studies have demonstrated a 


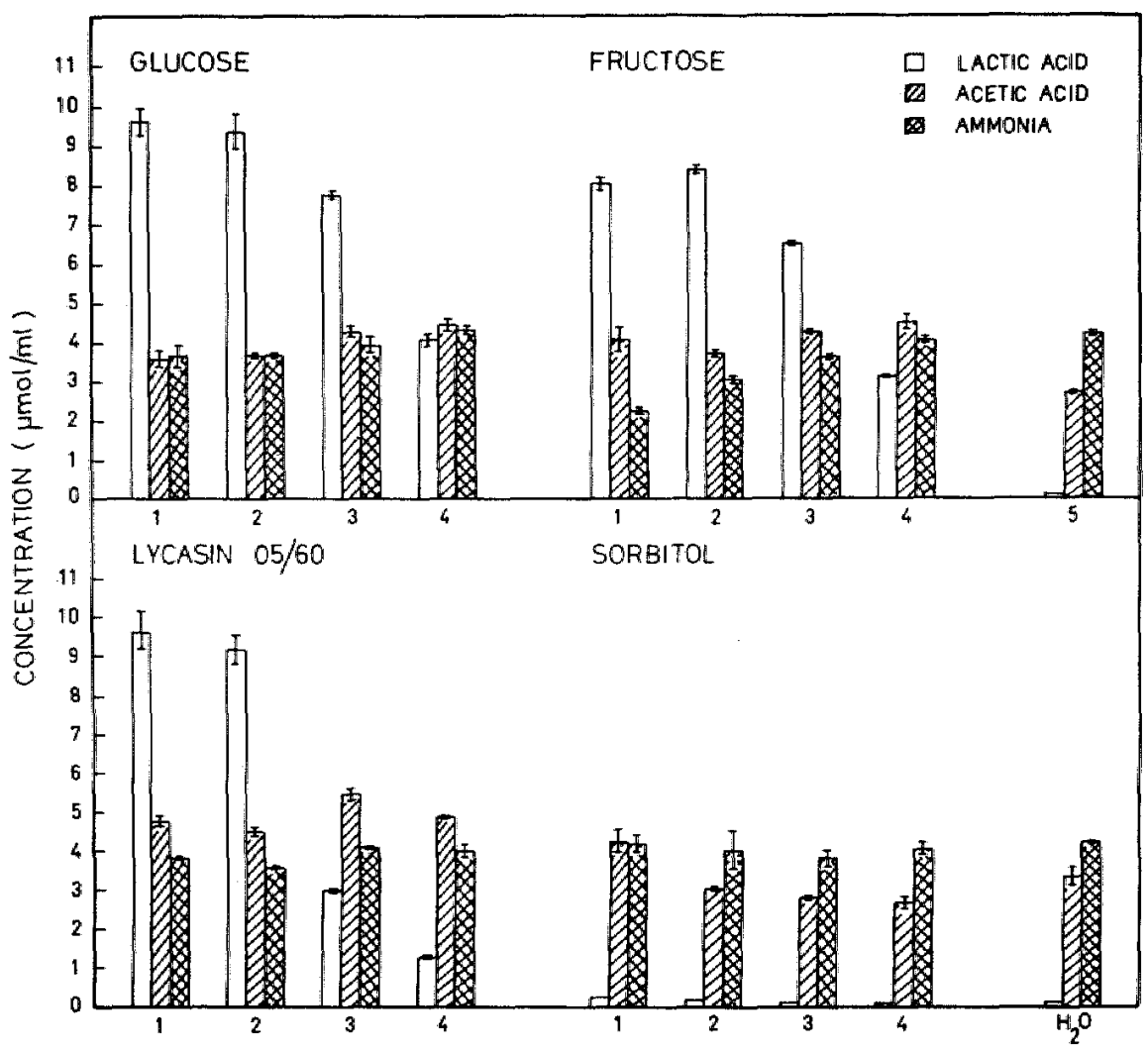

Fig. 2. The levels of lactic acid, acetic acid, and ammonia detected in the suspended salivary sediment mixtures after $4 \mathrm{~h}$ incubation in the presence of $10 \%$ ( $w / v)$ mixtures of xylitol and other carbohydrates. The columns show the means and ranges of duplicate determinations. For other details, see Fig. 1.

very slight or no inhibitory effect on acid production by xylitol in mixtures of sucrose and xylitol $(12,17,20)$. Our results are in agreement with the latter ones, suggesting that xylitol exerts no clear inhibitary effect on acid production of mixed oral flora when used in combination with easily fermentable carbohydrates. The ratio of xylitol to the fermentable carbohydrate in the mixture was of little importance.

It has been previously shown that the slow acid production from sorbitol in plaque is further decreased by the presence of $x y l i t o l$ (18). This decrease has been attributed to the formation of xylitol-5-phosphate in some oral bacteria, for example S. mutans, this compound being toxic to these bacteria (19). In the present study, an increase in $\mathrm{pH}$ values in the control mixtures containing no added carbohydrate or xylitol alone was found only in the xylitol-sorbitol mixtures, independent of the ratio of xylitol to sorbitol involved. Thus, out of the five sucrose substitutes and their mixtures tested in this study, 
only those of sorbitol and xylitol were associated with an increase of the $\mathrm{pH}$ values of mixed oral flora.

The lactic acid production alone seemed to explain the $\mathrm{pH}$ decreases observed in the mixed oral flora, a result which is in agreement with earlier findings $(21,22)$. The relatively similar levels of acetic acid found with most carbohydrates and the control mixtures suggest that this acid was mainly generated from endogenous sources. Also, the similar levels of ammonia detected after all incubations reflected the urea concentration of the whole saliva used in the incubations, rather than a specific effect of the carbohydrates on the base metabolism of the mixed oral flora. Though acetic acid has been proposed to have buffering properties under the conditions prevailing in the oral cavity (23), the ratio of acetic acid to lactic acid seerned to be of minor importance as a $\mathrm{pH}$-regulating factor under the conditions of this study. The ratio of ammonia to acids (lactic acid and acetic acid) being above one, however, resulted in an increase in the $\mathrm{pH}$ values observed in the three xylitol-sorbitol mixtures, in those containing xylitol alone, and in the controls containing no added carbohydrate.

The beneficial effects of xylitol on oral health are mainly based on the nonfermentability of this polyol by most oral bacteria (8), although physicochemical effects may also contribute to the final outcome $(24,25)$. Thus, according to the present and other studies $(17,18)$, one of the most important mechanisms of the action of xylitol will be abolished if xylitol is mixed with easily fermentable carbohydrates, such as fructose, glucose or sucrose. In mixtures with Lycasin 80/55, which is fermented slowly but to a greater extent than sorbitol (11), addition of xylitol did not lead to an increase in the $\mathrm{pH}$ values during incubation; however, the $\mathrm{pH}$ drops were very small. Thus, in mixtures with sorbitol and Lycasin 80/55, xylitol should promote remineralization as ef- ficiently as when used alone. However, the possible adaptation of the oral flora must be taken into consideration when using slowly fermentable carbohydrates in mixtures with xylitol (26). For xylitol alone such adaptation has not been reported (27).

Although maximization of the beneficial pH effects of xylitol would naturally presuppose that these polyols should not be mixed with other carbohydrates in consumer products, it appears that those effects should be retained in mixtures of xylitol and certain slowly fermented carbohydrates, such as sorbitol and Lycasin 80/55.

Acknowledgment - The financial support of the Research Foundation of Finnish Sugar Co. Ltd. is gratefully acknowledged.

\section{References}

1. Gehrung $\mathbb{F}$, Mäkingn $\mathrm{K}$, Larmas M, ScheiNEN A. Turku sugar studies. X. Occurrence of polysaccharide-forming streptococci and ability of the mixed plaque microbiota to ferment various carbohydrates. Acta Odontal Scand 1975; 33: Suppl. 70: 223-37.

2. Edwardsson S, Birkhed D, Mejare B. Acid production from Lycasin, maltitol, sorbitol and xylitol by some oral streptococci and lactobacilli. Acta Odontol Scand 1977; 35: 257-63.

3. Havenaak R, Huis in'T Veld JHI, Backer Drrks O, de StoppelaAr JD. Some bacteriological aspects of sugar substitutes. In: Guggenherm B, ed. Health and sugar substitutes. Proceedings from ERGOB Conf., Geneva, 1978. Basel: Karger, 1979; 192.

4. Pelatt D, Werrin, SR. Acid production from alditols by oral streptococci. $f$ Dent Res 1979 ; 58: $1733-4$

5. Gehring F, Märinen $\mathbb{K}$, Larmas $M$, SchedNIN A. Turku sugar studies. IV. An intermediate report on the differentiation of polysaccharide-forming streptococci (S. mutans). Acta Odontol Scand 1974; 32: 436-44.

6. Loesche WJ, Grossman NS, Earnest R, CorPRON $R$. The effect of chewing xylitol gum on the plaque and saliva levels of Streptococcus mutans. JADA 1984; 108: 587-92. 
7. Leach SA, Agalamanyt EA, Green KM. Remineralization of the teeth by dietary means. In: Demineralization and remineralization of the teeth, London: IRL Press Ltd., 1983; 57-73.

8. Märinen KK, Saheinen A. Xylitol and dental caries. Ann Rev Nutr 1982; 2: 133-50.

9. Kleingerg I, Graw D, Komirama $K$. Effect of salivary supernatant on the glucolytic activity of the bacteria in salivary sediment. Arch Oral Biol 1973; 18: 787-98.

10. Pakkala U, Ligsmaa $H$, Mähinen KK. Use of xylitol in the control of oral hygiene in mentally retarded children. Proc Finn Dent Soc 1981; 77. 271-7.

11. Von Gehring F, Hufnagei. H-D. Intra- und extraorale $\mathrm{pH}$-Messungen an Zahnplaques des Menschen nach Spülungen mit einigen Zucker- und Saccharoseaustauschstoff-lösunger. Oralprophylaxe 1983; 5: 13-9.

12. Mäkinen KK. Microbial growth and metabolism in plaque in the presence of sugar alcohols. In: Stiles HM, Lotsche WJ, O'Brien $\mathrm{TG}$, eds. Microbial aspects of deatal caries. Spec. Suppl. Microbiol Abstr 1976; II: 263-76.

13. Mühlemann HR, Schmid R, Noguchi T, ImFELD T, Hirsch RS. Some dental effects of xylitol under laboratory and in in vivo conditions. Caries Res 1977; 11: 263-76.

14. Knuttila MLE, MäkineN KK. Effect of xylitol on the growth and metabolism of Streptococcus mutans. Caries Res 1975; 9: 177-89.

15. Vadeboncoeur C, Traman L, Mouton C, Mayrand D. Effect of xylitol on the growth and glycolysis of acidogenic oral bacteria. $\exists$ Dent Res 1983; 62. 882-4.

16. Kleber GJ, Schimmele RJ, Putt MS, Murler JC. The effect of tablets composed of various mixtures of sugar alcohols and sugars upon plaque $\mathrm{pH}$ in children. 7 Dent Res 1979; 58: $614-8$.
17. Drucker DB, Verran J. Comparative effects of the substance-sweeteners glucose, sorbitol, sucrose, xylitol and trichlorosucrose on lowering the $\mathrm{pH}$ by two oral Streptocaccus mutans strains in vitro. Arch Oral Biol 1980, 24: 965-70.

18. Frostell G. Interaction between xylitol and sorbitol in plaque metabolism. Sreed Dent 7 1984; 8: $137-46$.

19. Wáler SM, Rölla G, Assev S, Cardi JE. The effect of xylitol on plaque metabolism. Sweed Dent 7 1984; 8: 155-61.

20. Brasy BG, Fu J. Changes in plaque $\mathrm{pH}$ in vitro by sweeteners. F Dent Res 1985; 64: $1130-3$.

21. Grodes DAM. Acids produced by human dental plaque metabolism in situ. Caries Res 1975; 9: 98-109.

22. VRatsanos SM, Mandel ID. Effect of sucrose and hexitol-containing chewing gurns on plaque acidogenesis in vivo. Pharmacol Ther Dent 1981; 6: 87-91.

23. Margolis HC, Moreno EC, Murphy Bj. Importance of high $\mathrm{pKa}$ acids in cariogenic potential of plaque. 7 Dent Res 1985; 64: $786-92$

24. MÄKINEN KK, SöDERLING E. Solubility of calcium salts, enamel, and hydroxyapatite in aqueous solutions of simple carbohydrates. Cataf Tissue Int 1984; 36: 64-71.

25. Mäkinen KK. New biochemical aspects of sweeteners. Int Dem 7 1985; 35: 23-35.

26. Birkfied D, Edwardsson S, Kalfas S, SvenSÄTER G. Cariogenicity of sorbitol. Sued Dent 7 1984; 8:147-54.

27. Mäkimen KK, Söderling E, HämäLäINEN M, ANTonen P. Effects of long-term use of xylitol on dental plaque. Proc Finn Denl Soc 1985; 81: $28-35$. 
This document is a scanned copy of a printed document. No warranty is given about the accuracy of the copy. Users should refer to the original published version of the material. 